\title{
Diol Lipids in the Phospholipid Fraction of Lipomyces starkeyi Grown in the Medium Containing 1,2-Propanediol ${ }^{\dagger}$
}

\author{
Tetsuya Suzuki and Kiyozo Hasegawa \\ Research Institute for Food Science, Kyoto University, Uji, Kyoto, Japan
}

Received October 11, 1973

\begin{abstract}
After training for 1,2-propanediol, a yeast, Lipomyces starkeyi, grew well in the media containing the diol, showing a diauxie-like pattern. The yeast phospholipid was converted into monoacetyl-diglyceride by acetolysis. The presence of monoacetyl-mono fatty acyl esters of the diol in this fraction was found by GC-MS. The data suggested that the diol was present as 2-palmitoyl-ester and 2-oleoyl-ester in the form of phospholipid analogue, in which phosphorus bound in 1-position of the diol. Molecular species of the phospholipid was also deduced from the GC-MS data. Diol diester was not found in the triglyceride fraction of the yeast.
\end{abstract}

Following the first discovery of a fatty acid ester of 2,3-butanediol, named as coixenolide, by Ukita et al.," several kinds of short chain diols, ethyleneglycol, propanediol, butanediol and so on, were detected in lipid hydrolyzates from many biological sources by many investigators." ${ }^{2}$ However, only one diol phospholipid had been reported until the recent studies by Bergelson et al. ${ }^{31}$ Little is known about the biogenesis of diol lipids. It has not been known whether a free diol in the medium would be incorporated into the lipid as an alcohol constituent or not.

The present investigation was undertaken to find out if the diol supplemented in the cultivation medium would be incorporated into the lipid of the yeast, L. starkeyi. During the course of this experiment, a direct method to distinguish the free diol from the diol lipids became necessary. To resolve this problem, acetolysis method and combined gas chromato-

$t$ A part of this work was presented at the Annual Meeting of the Agricultural Chemical Society of Japan, held in Tokyo, April, 1973, and also at 9th International Congress of Biochemistry, held in Stockholm, Sweden, July, 1973.

Abbreviations used: MADG, monoacetyl-diglyceride; C-M, chloroform-methanol; GC-MS, gas chromatography-mass spectrometry; TLC, thin-layer chromatography; TMS, trimethyl silylether; GLC, gas-liquid chromatography; DEGS, diethyleneglycol succinate polyester. graphy-mass spectrometry were employed. The results showed that diol lipids were detected in the phospholipid fraction obtained from the cells harvested at the stages adaptating and consuming 1,2-propanediol.

\section{MATERIAIS AND METHODS}

Microorganism and culture conditions. The microorganism used in this study was Lipomyces starkeyi IFO 0678. In order to adapt L. starkeyi to the new carbon source, it was preliminarily cultured in the following three different media. The first strain was. grown in a glucose medium, then transferred to the medium containing $1 \%$ of 1,2 -propanediol and $4 \%$ of glucose, and then transferred to $2.5 \%$ each of $1,2-$ propanediol and glucose. The strain cultivated in the last medium was used in the main culture.

The culture medium used in this experiment was as. follows. $\mathrm{D}-(+)$-Glucose, $2.5 \%$; $\mathrm{D}, \mathrm{L}-1,2$-propanediol, $2.5 \% ;\left(\mathrm{NH}_{4}\right)_{2} \mathrm{SO}_{4}, 0.5 \% ; \mathrm{KH}_{2} \mathrm{PO}_{4}, 0.1 \% ; \mathrm{MgSO}_{4}$. $7 \mathrm{H}_{2} \mathrm{O}, 0.05 \% ; \mathrm{CaCl}_{2} \cdot 2 \mathrm{H}_{2} \mathrm{O}, 0.001 \% ; \mathrm{NaCl}, 0.001 \%$; yeast extract, $0.1 \%$. Initial $\mathrm{pH}$ was adjusted to 6.4 . Cultivation was carried out at $30^{\circ} \mathrm{C}$ under shaking. During the cultivation, the $\mathrm{pH}$ of the medium was controlled at approximately 4 to 6 by means of adding $1 \mathrm{~N}-\mathrm{NaOH}$. Aliquots of yeast cells were harvested at $100 \mathrm{hr}, 121 \mathrm{hr}, 168 \mathrm{hr}$ and $237 \mathrm{hr}$. Harvested cells were lyophilized and stored at $-20^{\circ} \mathrm{C}$ until the analysis of lipids. During the cultivation, glucose content was determined by phenol-sulfuric acid method," and 1,2propanediol was determined by chromotrope-periodide method. ${ }^{51}$ TLC with "Avicel" was also used to detect glucose and 1,2-propanediol consumption. ${ }^{6}$ 


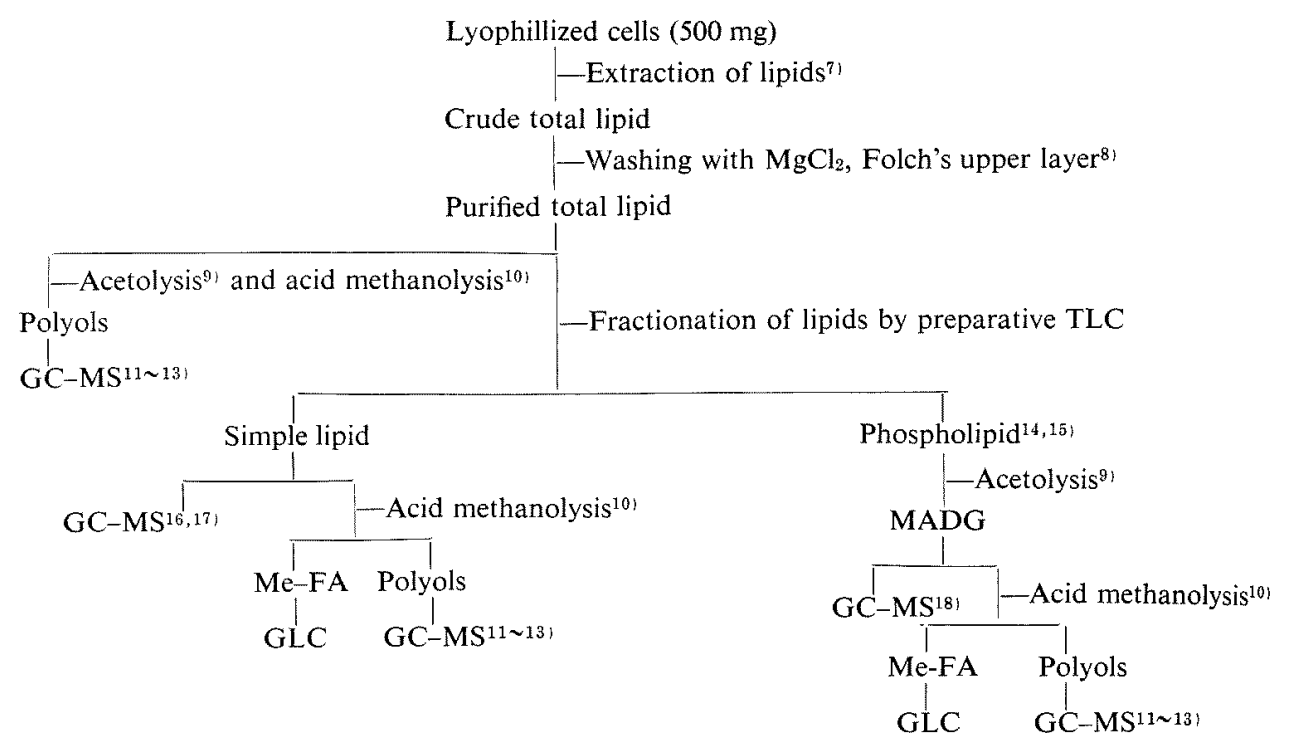

SCHEME 1. Experimental Procedure for the Diol Lipid Analysis.

Experimental procedure for diol lipid analysis. The analysis of diol lipid in the yeast lipid was carried out by the process shown in Scheme 1. Reagents used were guaranteed reagent grade. Methanol used in acid methanolysis was super dried.

i) Extraction of the lipid. Extraction of the lipid was carried out according to Pedersen's method, ${ }^{7 !}$ followed by washings with $1.5 \times 10^{-\frac{1}{4}} \mathrm{M}$ of $\mathrm{MgCl}_{2}$ solution once, and then with Folch's upper layer ${ }^{8}$ twice. These washings removed free 1,2-propanediol completely. From the total lipid, an aliquot was supplied for polyol analysis, and the rest was used for the fractionation of lipids.

ii) Analysis of polyols in the total lipid fraction. By acetolysis $^{97}$ of the total lipid fraction, phospholipids, monoglycerides and diglycerides were converted to acetyl-glycerides, while triglycerides remained unchanged. They were further converted into fatty acid methyl esters and free polyols by acid methanolysis. ${ }^{10}$ Free polyol fractions containing $\mathrm{HCl}$ were neutralized with Dowex $1 \times 4\left(\mathrm{OH}^{-}\right)$prior to GLC. Free polyols released were then analyzed by GC-MS as free forms, ${ }^{11}$, TMS-derivatives $^{12}$ and acetyl-derivatives. ${ }^{13}$

iii) Fractionation of total lipid extracts by preparative $T L C$. Total lipid fractions were chromatographed on the plates of silica gel $\mathrm{H}$ (Merck, $500 \mu$ thick) using petroleum ether-ethyl ether $(85: 15, \mathrm{v} / \mathrm{v})$ in a saturation tank. Zones corresponding to sterol ester, triglyceride, free fatty acid, diglyceride and free sterol were combined and then extracted by means of C-M $(2: 1, v / v)$ and petroleum ether. The combined extract was referred to as "simple lipid." Zones corresponding to mono- glyceride and origin were rechromatographed to separate the phospholipids from the monoglyceride by using silica gel $\mathrm{H}$ ( $500 \mu$ thick) developed with $\mathrm{C}-\mathrm{M}-\mathrm{NH}_{4} \mathrm{OH}(65: 35: 5, \mathrm{v} / \mathrm{v})$ for $5 \mathrm{~cm}$. Phospholipids were recovered by C-M $(2: 1, v / v)$ extraction. The content of the phospholipids were determined according to the method of Bartlett, ${ }^{14}$ and the identification of the phospholipid species were performed by TLC. ${ }^{15 i}$

iv) $G C-M S$ of simple lipid. $\mathrm{GC}-\mathrm{MS}$ analysis of simple lipid was carried out on a gas chromatographmass spectrometer (Shimadzu LKB-9000) equipped with a $350 \mathrm{~mm} \times 3 \mathrm{~mm}$ column packed with $2.5 \%$ OV-17 on Shimalite W (60 80 mesh). The column temperature was raised from 200 to $320^{\circ} \mathrm{C}$ at a rate of $3^{\circ} \mathrm{C} / \mathrm{min}$. The injector temperature was kept at $310^{\circ} \mathrm{C}$, and molecular separator was at $330^{\circ} \mathrm{C}$. The temperature of the ion source was $350^{\circ} \mathrm{C}$. The flow rate of the carrier gas, $\mathrm{He}$, was controlled at $30 \mathrm{ml} / \mathrm{min}$. The ionizing electron energy amounted to $70 \mathrm{eV}$, the trap current to $60 \mu \mathrm{A}$, the accelerator voltage to $3.5 \mathrm{kV}$, and slits were $0.1 \mathrm{~mm}$. The scanning limit was $0 \sim 1000$ $m / e$. The GLC conditions used were an application of Kuksis' triglyceride separation by GLC. ${ }^{16 \mid}$ Mass spectra obtained were interpreted according to the finding of Barber et al. ${ }^{17 !}$

v) GC-MS of MADG. Phospholipids were converted to MADG by acetolysis ${ }^{9 /}$ and then analyzed by GC-MS. ${ }^{18:}$ The experimental conditions of GC-MS were the same as described above, except the temperature of the column oven which was raised from 180 to $290^{\circ} \mathrm{C}$

vi) GLC of fatty acid methyl ester. Fatty acid methyl esters were prepared by acid methanolysis, ${ }^{10\}}$ 
and then analyzed by a gas chromatograph (Shimadzu GC-5A) using a column packed with $15 \%$ DEGS on Neopak AS $\left(60 \sim 80\right.$ mesh) at $187^{\circ} \mathrm{C}$ under $\mathrm{N}_{2}$ flow at $60 \mathrm{ml} / \mathrm{min}$.

Chemical synthesis of monoacetyl palmitoyl propanediol. Monoacetyl palmitoyl propanediol was prepared by acetolysis of monopalmitoyl propanediol which was chemically synthesized according to the method of Baer et al. ${ }^{19}$ )

\section{RESULTS}

Growth curve of L. starkeyi in the medium containing 1,2-propanediol

Growth curve of $L$. starkeyi is shown in Fig. 1. As shown in Fig. 1, the consumption of 1,2-propanediol was much slower than that of glucose, and the growth curve followed a diauxie-like pattern. At the time indicated by arrows, yeast cells were harvested to analyze the diol lipids.

Qualitative and quantitative lipid analysis

Total lipid contents at different growth stages were $28.2,20.7,24.6$ and $8.7 \%$ at 100 , 121, 168 and $237 \mathrm{hr}$, respectively. Phospholipid contents were $3.9,2.9,6.5$ and $13 \%$ at the
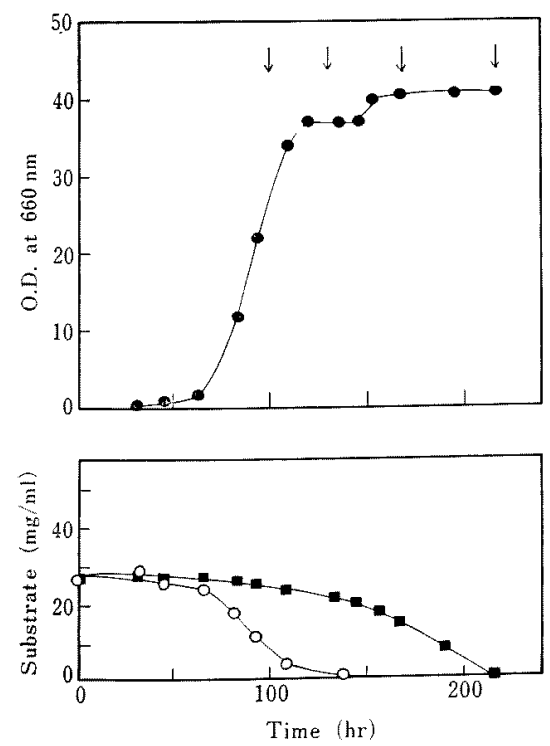

FIG. 1. Growth Curve and Carbon Source Consumption of L. starkeyi.

--D, turbidity at $660 \mathrm{~nm}$; - - 1,2-propanediol content $(\mathrm{mg} / \mathrm{ml}) ; 0-0$, glucose content $(\mathrm{mg} / \mathrm{ml})$. stages described above, respectively.

The lipid composition analyzed by TLC consisted of sterol-ester, triglyceride, free fatty acid, diglyceride, free sterol, probably ergosterol, monoglyceride (trace) and phospholipid. Among these compounds, triglycerides occupied $70 \sim 80 \%$ of the total lipid. The qualitative patterns of lipid did not change throughout the cultivation.

The phospholipid was composed of phosphatidylethanolamine, phosphatidylcholine, phosphatidylserine, phosphatidylinositol and a small amount of phosphatidylglycerol. Although the content of an individual phospholipid component altered by the growth stages, the kind of phospholipids did not change.

Diol in the polyol fraction of total lipids

By means of GC-MS, appreciable amounts of 1,2-propanediol were detected in the polyol

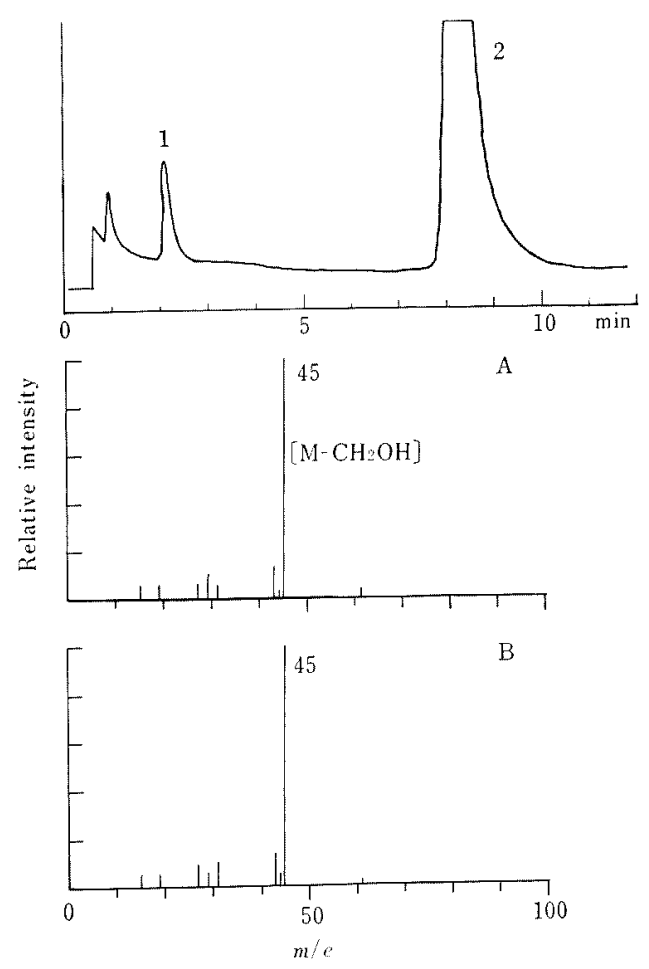

Fig. 2. Gas Chromatogram and Mass Spectra of Polyols in the Total Lipid of $168 \mathrm{hr}$ Yeast.

Peak 1, 1,2-propanediol; peak 2, glycerol. A, peak 1; $\mathrm{B}$, authentic 1,2-propanediol. 
fraction of the total lipids from the yeast cells cultivated for 100,121 and $168 \mathrm{hr}$, respectively (Fig. 2). The free polyol fraction from $168 \mathrm{hr}$ cultured yeast cells contained the highest content of 1,2-propanediol $(0.3 \%$ of total polyols). The three samples described above were further fractionated to simple lipids and phospholipids in order to investigate in which fraction the diol lipid was contained.

\section{GC-MS analysis of simple lipid fractions}

By means of GC-MS as described above, simple lipid fractions of 100,121 , and $168 \mathrm{hr}$ cultivated yeast cells were analyzed, however, no diol lipid was detected. Fatty acid species of triglycerides were analyzed by GC-MS, and it was found that dioleoyl-palmitoylglycerol (monopalmitoyl-diolein) was the major triglyceride throughout the stages examined.

No diol was detected in the analysis of the polyol fractions of simple lipid.

\section{GC-MS analysis of $M A D G$ fraction from phospholipids}

Phospholipid fractions of the 100,121 , and $168 \mathrm{hr}$ yeast cells were submitted to acetolysis, and then the MADG fractions were analyzed by GC-MS. The gas chromatogram and mass spectra are shown in Figs. 3 and 4. Interpretations of individual mass spectra were carried out according to the method of Hasegawa et al., ${ }^{18}$ which was based on the triglyceride analysis of Barber et al. ${ }^{17}$ According to their principle, the fatty acid moieties were identified

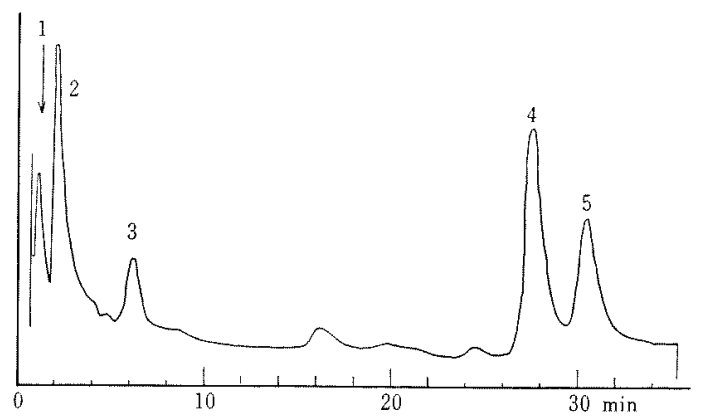

FIG. 3. Gas Chromatogram of MADG Fraction of 168 hr Yeast. by the intense peaks corresponding to the $[\mathrm{M}-\text { acyloxy }]^{+}$ion, and to the acylium ion peaks. The fatty acid in the 2-position of the glycerol was identified from the [M-acyloxymethylene $]^{+}$peaks. The [acylium +74$]^{+}$and $[\text { acylium }+128]^{+}$peaks were taken as a proof of the direct attaching of the acid to the glycerol skelton. The peaks at 43,117 and 171 , corresponding to $\left[\mathrm{CH}_{3} \mathrm{CO}\right]^{+},\left[\mathrm{CH}_{3} \mathrm{CO}+74\right]^{+}$and $\left[\mathrm{CH}_{3} \mathrm{CO}+128\right]^{+}$, respectively, are characteristic ones of MADG. The peak at 159 derived from $[\mathrm{M}-\text { acyloxy }]^{+}$ion is a characteristic peak of the mass spectrum of diacetyl monoglyceride.

The mass spectrum of monoacetyl monoacyl propanediol also gave an acylium ion, an $[\mathrm{M} \text {-acyloxy }]^{+}$ion, and an [M-acyloxymethylene $]^{+}$ion, yet the mass spectrum of monoacetyl monoacyl propanediol was easily distinguished from that of glycerophospholipids, because the former lacked [acylium +74$]^{+}$and $[\text { acylium }+128]^{+}$ions and had a characteristic peak at 101 corresponding to [M-acyloxy $]^{+}$ ion.

A, B, C, D and E in Fig. 4 show the mass spectra of the peaks 1, 2, 3, 4 and 5 in Fig. 3, respectively.

In the spectrum of $\mathrm{D}$, the peaks at $m / e 43$ and 117 and the acylium ions at 239 and 265 suggested the structure of a palmitoyl-oleoylacetylglycerol. From the fragments $[\mathrm{M}-$ acyloxy $]^{+}$at 381 and 355 and [M-acyloxymethylene $]^{+}$at 367 , this compound was identified as 1-palmitoyl-2-oleoyl-3-acetyl-glycerol.

Similarly the compound at peak 5 was identified as 1,2-dioleoyl-3-acetyl-glycerol.

In the mass spectrum of peak 1 eluted at $183^{\circ} \mathrm{C}$, the peaks at $m / e 43$ and 101 indicated that the compound was an acetylated compound of 1,2-propanediol, and the peak at m/e 239 was the acylium ion peak due to palmitoyl residue. Therefore, the compound at peak 1 was supposed to be an acetyl-palmitoylpropanediol. The found values agreed with the theoretical values of $m / e 356$ for molecular ion, $m / e 101$ for $\left[\mathrm{M}-\right.$ acyloxy] ${ }^{+}$ion and $m / e 297$ for $[\mathrm{M}-\text { acetoxy }]^{+}$ion. The peak at $m / e 283$ corresponded to the fragment [M-acetoxy- 


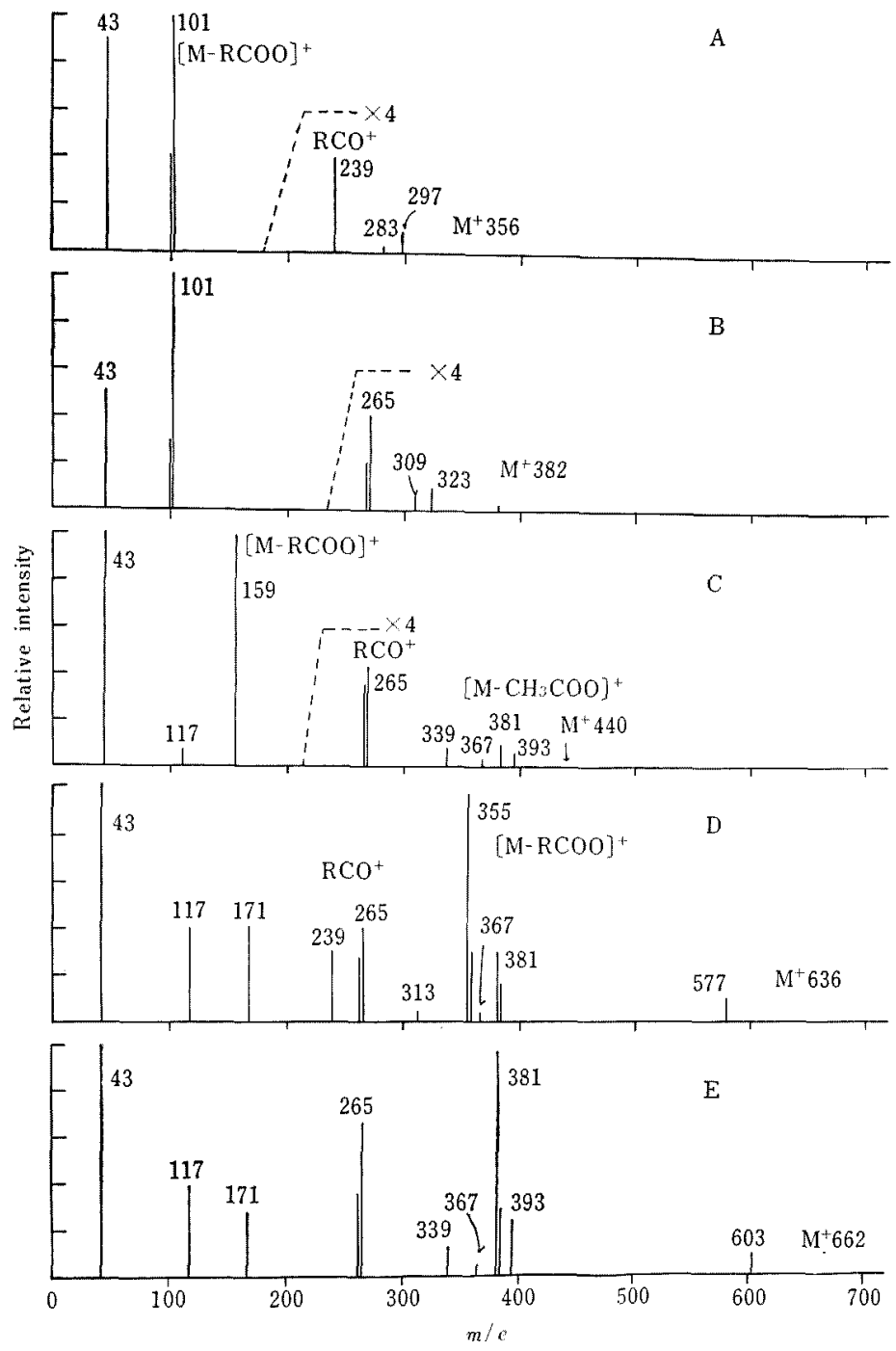

FIG. 4. Mass Spectra of Acetylated Derivatives in MADG Fraction of $168 \mathrm{hr}$ Yeast.

$\mathrm{M}^{+}$, molecular ion; $\mathrm{RCO}^{+}$, acylium ion; $\mathrm{RCOO}$, acyloxy residue.

A, peak 1, 1-acetyl-2-palmitoyl-propanediol; B, peak 2,1-acetyl-2-oleoyl-propanediol; C, peak 3, 2-oleodiacetin; D, peak 4, 1-palmitoyl-2-oleoyl-3-acetyl-glycerol; E, peak 5, 1,2-dioleoyl-3-acetylglycerol.

nethylene $]^{+}$ion. Finally the compound at leak 1 was identified as 1-acetyl-2-palmitoylropanediol.

Similarly the compound at peak 2 eluted it $190^{\circ} \mathrm{C}$ was identified as 1-acetyl-2-oleoylropanediol.

The structure of the compound at peak 1 vas confirmed further by direct comparison vith a synthetic sample of 1-acetyl-2-palmitoylropanediol. As shown in Fig. 5, the mass spectrum of the synthetic sample agreed with that of peak 1 .

In the interpretation of the mass spectrum of peak 3 eluted at $196^{\circ} \mathrm{C}$, the retention time of which on GLC was similar to that of an monoacetyl monoacyl diol, however it was found from the MS data that the peak was not monoacetyl monoacyl diol but diacetyl monoacyl glycerol. The peaks at $m / e 43,117$ and 171 suggested that the structure was an acetylated 

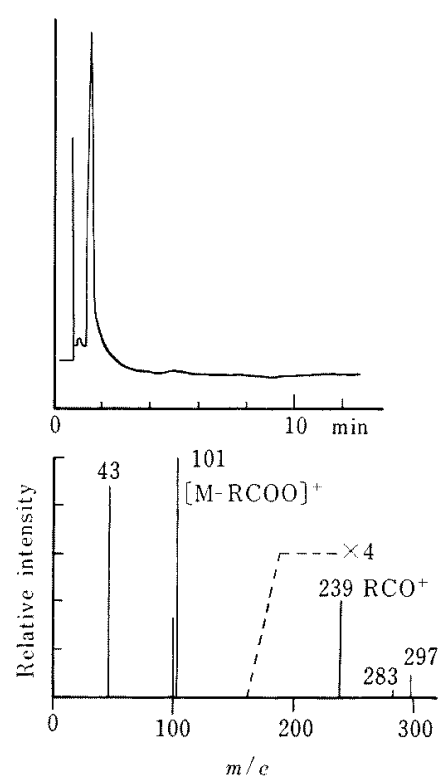

FIG. 5. Gas Chromatogram and Mass Spectrum of Synthetic 1-Acetyl-2-palmitoyl-propanediol.

compound of glycerol. The most intense peak at $m / e 159$ and acylium ion at $m / e 265$ suggested the structure of an oleoyl-diacetyl-glycerol. The found values agreed with the theoretical values of $m / e 440$ for molecular ion and $m / e$ 159 for $[\mathrm{M}-\text { acyloxy }]^{+}$ion. Since $[\mathrm{M}-$

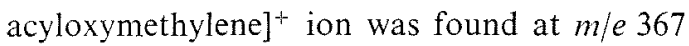
and not at $m / e 145$, peak 3 was identified as 2oleo-diacetin.

In the acetolysis product obtained from the sample of $100 \mathrm{hr}$, trace amount of 1-acetyl-2oleoyl-propanediol was detected. In the sample of $121 \mathrm{hr}$, 1-acetyl-2-linoleoyl-propanediol which was eluted at $190^{\circ} \mathrm{C}$ and 1-acetyl2-oleoyl-propanediol were detected. However, the contents of both compounds were only trace.

\section{Polyols and fatty acids from the $M A D G$ fraction}

The polyols of the MADG fraction were liberated by means of acid methanolysis and analyzed by GC-MS. Figure 6 shows a gas chromatogram of polyols liberated from the MADG fraction of the sample at $168 \mathrm{hr}$. By the interpretation of mass spectra, the small peak eluted at $3.5 \mathrm{~min}$ was identified as 1,2-
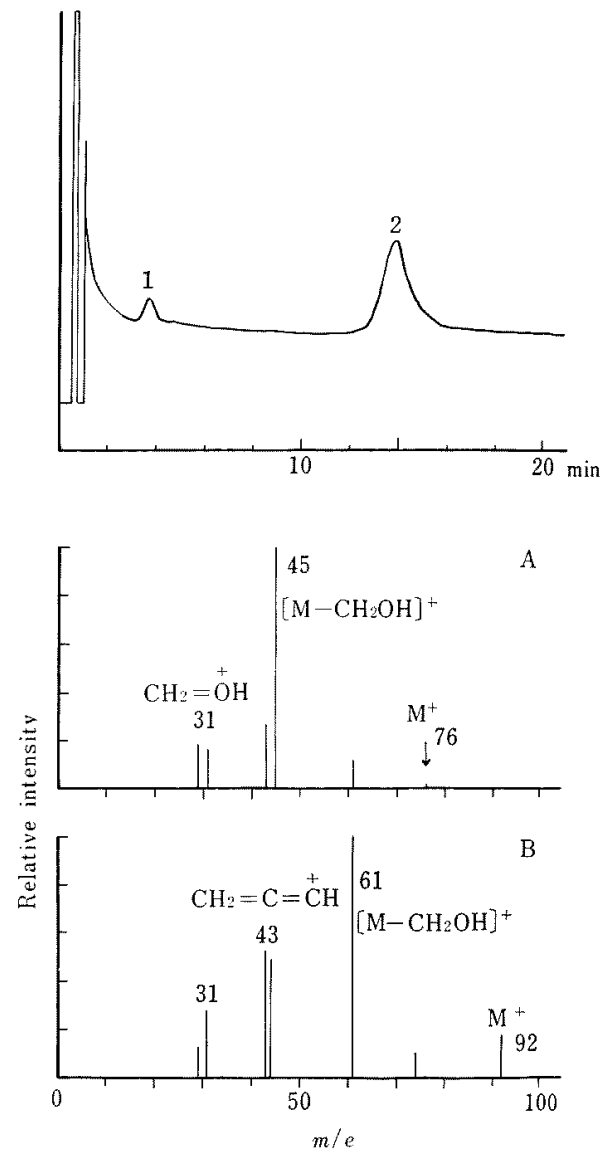

Fig. 6. Gas Chromatogram and Mass Spectra of Polyols Liberated from the Acetylated Derivatives of Phospholipid of $168 \mathrm{hr}$ Yeast.

A, peak 1, 1,2-propanediol; B, peak 2, glycerol.

propanediol, and the peak at $14 \mathrm{~min}$ as glycerol. The approximate content of 1,2-propanediol in the polyol fraction from the phospholipid was about $2.4 \%$.

Fatty acid composition analyzed by GLC showed that major fatty acids were oleic acid $(50.2 \%)$ and palmitic acid $(18.7 \%)$, while palmitoleic acid $(6.6 \%)$ and linoleic acid $(7.3 \%)$ were minor components.

\section{Molecular species of $M A D G$}

The major species found in the MADG derived from the phospholipid fractions from samples of 100 and $121 \mathrm{hr}$ yeast cells were 1-palmitoyl-2-oleoyl type and 1-oleoyl-2linoleoyl type. The major compound found 
in a $168 \mathrm{hr}$ sample was 1-palmitoyl-2-oleoyl type. However, from the $237 \mathrm{hr}$ sample, the major compound found was 1,2-dioleoyl type.

\section{DISCUSSION}

Ethyleneglycol, propanediols, butanediols and other diols had been detected in many biological sources. However indirect methods were usually used to detect these diol lipids. With the indirect methods in which lipids are hydrolyzed to release free polyols, there is a possibility that free diols may be recognized to be diol lipids. In fact we found that, by the usual methods, the free 1,2-propanediol contaminated from the medium may be recognized as diol lipid.

In this study, a new direct identification method of diol phospholipids is reported. It is based on the same principle as the analysis of MADG by GC-MS reported previously. ${ }^{18)}$ By means of acetolysis, glycerophospholipids are converted into MADG and diol-phospholipids into monoacetyl monoacyl diol. The acetylated derivatives are easily separated by GLC on the silicone column according to the sum of the carbon numbers of fatty acyl residues, and then the structure of monoacetyl monoacyl diol is interpreted by means of the mass spectrometry. In the case of 1,2-propanediol, the peak at $m / e 101$ corresponding $[\mathrm{M}-\text { acyloxy }]^{+}$ion and the peak corresponding $[\mathrm{M} \text {-acetoxy }]^{+}$ion were the direct evidence of monoacetyl monoacyl propanediol.

As reported by Bergelson, ${ }^{2}$ several kinds of diol phospholipids dissolve considerably in water, thus repeated washing is not recommended. In such a case, the present method, in which the diol phospholipid is analyzed in a form of acetyl derivative, would be an effective method to distinguish the free diol from the lipid diol. Thus if the free diol is contaminated in the phospholipid fraction, it is converted into diacetyl diol by acetolysis, and this compound is easily distinguished from the long chain fatty acid ester of monoacetyl diol, since the characteristic peak of $\mathrm{RCO}^{+}$lacks in the spectrum of the diacetyl diol.
Few reports have been presented concerning the biogenesis of diol lipids. The results we have obtained may offer the data suggesting that, if free diols are given, they will become incorporated into the alcohol component of the lipid. However contrary to our expectation, a diol lipid was not detected in the simple lipid fraction but in the phospholipid fraction. Our results would be the first one that diol lipid was found in the phospholipid fraction of yeast, though Bergelson and his coworkers recently reported the finding of ethyleneglycol plasmalogen in a rat liver. ${ }^{3}$ It is now under the investigation which type of base was bound to the diol phospholipid. The mechanism of the diol lipid synthesis in nature is still unknown, thus it is necessary to have further studies on the conditions under which the diol lipid is synthesized.

Changes in the fatty acid moieties in the triglycerides and phospholipids during cultivation were also examined by means of GC-MS, and some interesting results were obtained. The data on this subject will be published elsewhere.

Acknowledgement. The authors wish to express their thanks to Prof. K. Iwai, Research Institute for Food Science, Kyoto University, for his kind advice and encourgement. They also indebted to Prof. T. Tochikura, Kyoto University for supplying the yeast and to Prof. H. Yamada, Kyoto University, for his kind advice.

\section{REFERENCES}

1) T. Ukita and A. Tanimura, Chem. Pharm. Bull. (Tokyo), 9, 43 (1961).

2) L. D. Bergelson, "Progress in the Chemistry of Fats and Other Lipids," Vol. X, ed. by R. T. Holman, Pergamon Press, Oxford, 1970, pp. $239 \sim$ 286.

3) L. D. Bergelson, V. A. Vaver, N. V. Prokazova, A. N. Ushakov, B. V. Rozynov, K. Stefanov, L. I. Ilukina and T. N. Simonova, Biochim. Biophys. Acta, 260, 571 (1972).

4) M. Dubois, K. A. Gilles, J. K. Hamilton, P. A. Rebers and F. Smith, Anal. Chem, 28, 350 (1956).

5) M. A. Wells and J. C. Dittmer, Biochemistry, 4, 2459 (1965).

6) D. W. Vomhof and T. C. Tucker, J. Chromatog., 17, 300 (1965). 
7) T. A. Pedersen, Acta Chem. Scand, 16, 374 (1962).

8) J. Folch, M. Less and G. H. Sloan Stanley, J. Biol. Chem., 226, 497 (1957).

9) O. Renkonen, J. Amer. Oil. Chem. Soc., 42, 298 (1965).

10) W. Stoffel, W. Insull and E. H. Ahrens, Anal. Chem., 31, 307 (1959).

11) T. Suzuki and K. Hasegawa, Nippon Nogeikagaku Kaishi, 46, 215 (1972).

12) K. Hasegawa, M. Murata and T. Suzuki, Yukagaku, 21, 35 (1972).

13) E. Jellum and P. Björnstadt, J. Lipid Res., 5, 314 (1964).
14) G. R. Bartlett, J. Biol. Chem., 234, 466 (1959).

15) W. D. Skidmore and C. Enteman, J. Lipid Res., 3, 47 (1962).

16) A. Kuksis, J. Amer. Oil Chem. Soc., 42, 269 (1965).

17) M. Barber and T. O. Merren, Tetrahedron Letters, 18, 1063 (1964).

18) K. Hasegawa and T. Suzuki, Lipids, 8, 631 (1973); idem, Abstracts of Papers, 20th Meeting of Kinki Branch of Japanese Biochemical Society, Sakai, Osaka, May, 1973, p. 42.

19) E. Baer, A. J. Duke and D. Buchnea, Can J. Biochem., 46, 69 (1968). 